\title{
Review Article \\ Current Progress in the Rejuvenation of Aging Stem/Progenitor Cells for Improving the Therapeutic Effectiveness of Myocardial Repair
}

\author{
Gurleen Kaur and Chuanxi Cai $\mathbb{D}$ \\ Center for Cardiovascular Sciences, Department of Molecular and Cellular Physiology, \& Department of Medicine, Albany Medical \\ College, Albany, NY 12208, USA \\ Correspondence should be addressed to Chuanxi Cai; caic@mail.amc.edu
}

Received 20 September 2017; Accepted 13 March 2018; Published 29 March 2018

Academic Editor: Shimon Slavin

Copyright (c) 2018 Gurleen Kaur and Chuanxi Cai. This is an open access article distributed under the Creative Commons Attribution License, which permits unrestricted use, distribution, and reproduction in any medium, provided the original work is properly cited.

Ischemic heart disease affects a majority of people, especially elderly patients. Recent studies have utilized autologous adult stem/ progenitor cells as a treatment option to heal cardiac tissue after myocardial infarction. However, donor cells from aging patients are more likely to be in a senescent stage. Rejuvenation is required to reverse the damage levied by aging and promote a youthful phenotype. This review aims to discuss current strategies that are effective in rejuvenating aging cardiac stem cells and represent novel therapeutic methods to treat the aging heart. Recent literature mainly focuses on three approaches that aim to reverse cardiac aging: genetic modification, pharmaceutical administration, and optimization of extracellular factors. In vitro genetic modification can be used to overexpress or knock down certain genes and allow for reversal of the aging phenotype. Pharmaceutical administration is another approach that allows for manipulation of signaling pathways related to cell proliferation and cell senescence. Since the stem cell niche can contribute to the age-related decline in stem cell function, rejuvenation strategies also include optimization of extracellular factors. Overall, improving the intrinsic properties of aging stem cells as well as the surrounding environment allows these cells to adopt a phenotype similar to their younger counterparts.

\section{Introduction}

Cardiovascular disease is the leading cause of mortality in the United States [1], and its risk increases in patients 65 years of age or older [2]. As the heart ages, the myocardium undergoes degeneration that leads to myocyte death [3]. Previous experiments conducted in the heart have explored whether the adult myocardium contains an undifferentiated pool of cells that may participate in cardiac repair [2]. The heart was initially thought to be a postmitotic organ without the capacity to replace itself. However, recent discoveries represent a major paradigm shift, suggesting that apoptotic cardiac cells are replaced by new cells derived from cardiac stem/progenitor cells (CPCs) [4]. Evidence has been obtained in favor of the regeneration of the aging myocardium. In a recent study, injection of autologous CPCs decreased scar size, increased the amount of visible myocardium, and improved regional function of the infarcted myocardium [5]. Local stimulation of CSCs can reverse the detrimental effects of aging on the heart and therefore represents a novel strategy for solving the problem of heart failure in the older population [2].

Obstacles to the success of stem cell-based clinical therapies include the poor survival of donor cells along with the age-related loss of stem cell regenerative capacity. More than $90 \%$ of transplanted mesenchymal stem cells (MSCs) die within the first few days [6]. Aging leads to diminished proliferative and differentiative potential due to increased oxidative stress, mitochondrial dysfunction, and genome instability [7]. Telomeres, repetitive nucleotide sequences at the ends of mammalian chromosomes that preserve chromosome stability and integrity, decrease in length with aging [8]. Accumulation of damage and shortening of telomeres leads to cellular senescence-a state of irreversible growth arrest [9]. Senescent cells are characterized by the incapability to contribute to 
tissue repair and regeneration. Aging is also associated with reactive oxygen species (ROS) that are generated by the mitochondria $[3,10,11]$. Mitochondrial overproduction of ROS also likely contributes to cellular senescence; it leads to the formation of highly reactive products $\mathrm{O}_{2}$ or $\mathrm{H}_{2} \mathrm{O}_{2}$, whose accumulation promotes senescence, DNA mutations, inflammation, and cell death pathways [8]. ROS can be detoxified within the cell by antioxidants such as superoxide dismutase (SOD) catalase, glutathione peroxidase, peroxiredoxin, and sulfiredoxin. However, an increase in ROS levels can subsequently alter the cell's normal redox state and provoke oxidative stress [12]. Therefore, rejuvenation is required to reverse the damage imposed by aging to restore tissue and organ function and improve longevity.

This review is designed to highlight current work in the field of rejuvenation of aging cardiac stem cells. Studies in this field have focused on three different approaches, summarized in Figure 1. The first category of strategies uses genetic modification to overexpress or knock down certain genes in cardiac stem cells. Certain proteins are found to either increase or decrease in expression in aging organisms, suggesting that reversal of this change in expression may rejuvenate older cells to a youthful phenotype. The second strategy for rejuvenation uses pharmaceutical administration to reverse senescence by targeting signaling pathways associated with important cellular processes such as proliferation, apoptosis, and senescence. Finally, the third strategy for rejuvenation involves optimizing the extracellular factors in order to prevent senescence and promote a youthful phenotype. A wide variety of stem/progenitor cells have been transplanted to improve cardiac regeneration, including skeletal myoblasts, hematopoietic stem cells, embryonic stem cells, and induced pluripotent stem cells [13-15]. However, this review focuses mainly on resident/adult mesenchymal stem cells and cardiac stem/progenitor cells.

\section{Genetic Modification}

In vitro genetic modification of aging cardiac stem cells to enhance proliferation, survival, and commitment is an effective strategy to enhance stem cell function and ensure improved cardiac output. Pim-1, a conserved serine/threonine protein kinase [1], is increased in expression in response to injury and protects against myocardial infarction [16] with its antiapoptotic and proproliferative actions [17]. Pim-1 kinase expression is higher in fetal human cardiac progenitor cells (hCPCs) as compared to older hCPCs, which suggests a correlation between Pim-1 expression and youthful phenotypic characteristic [18]. Recent studies have utilized hCPCs isolated from heart failure patients; hCPCs engineered to overexpress Pim-1 result in increased cellular engraftment and differentiation with improved vasculature [19]. Genetic modification with Pim-1 has the capability to rejuvenate with enhanced proliferation, decreased senescence, and increased telomere length [18]. A recent study found that Pim-1 expression in the heart coincides with nucleostemin (NS), a nucleolar protein required for cell cycle progression and proliferation. Reduced NS levels are associated with increased senescence and aging [20]. In addition, modification through
Pim-1 is extremely powerful because of the recent study, in which Pim-1 targeted to the nucleus preferentially enhanced stem cell youthfulness associated with reduced senescence while Pim-1 targeted to mitochondria promoted increased interaction with antiapoptotic proteins such as Bcl-x [21]. These new findings suggest that organelle-specific overexpression of Pim1 may be utilized in a more personalized form of regenerative medicine based on the specific properties of the patient's hCPCs.

Literature points to the paracrine effects of MSCs as being predominately responsible for cardiac repair. Some cytokines and growth factors produced by MSCs have been shown to be vital for cardiac protection while others are harmful for heart recovery. Therefore, optimization of MSCs before transplantation is required to maximize cell survival [22]. In a recent study, optimization was done through genetic modification of Rap1, a modulator involved in the NF- $\kappa \mathrm{B}$ pathway (Figure 2). Factors from the NF- $\kappa \mathrm{B}$ pathway have important roles in regulation of mitochondrial ROS, DNA replication, cell survival, and inflammation [23]. BM-MSCs with deletion of Rap1 were more tolerant than normal BM-MSCs to hypoxia that is associated with reduced activation of NF- $\kappa \mathrm{B}$ activity. Also, BM-MSCs with deletion of Rap1 showed a better therapeutic efficacy; they were associated with reduced inflammation postmyocardial infarction and enhanced cell survival of cardiomyocytes [24]. Another pathway that can be subjected to genetic modification and is also a paracrine regulator is the NRG1-ERBB4 signaling pathway (Figure 2). NRG1 is an essential paracrine regulator of cell-cell communication through activation of ERBB4, which further activates the PI3K/Akt pathway [25]. MSCs engineered with increased ERBB4 expression significantly preserve heart functions accompanied with reduced infarct size and enhanced cardiomyocyte division. A cardioprotective effect is seen due to activation of Akt and Bcl-2, which protects from apoptosis [26].

Aging is accompanied by a general decline in mitochondrial function in all tissues. Mitochondria may contribute to stem cell maintenance through regulation of specific metabolites such as NAD+ [27], whose effects are mediated by sirtuin family of NAD-dependent enzymes [28]. In myocardial tissue, SIRT3 localizes to mitochondria [29], reduces levels of reactive oxygen species (ROS), and blocks cardiac hypertrophic response through activation of Foxo-dependent antioxidants, manganese superoxide dismutase (MnSOD), and catalase [30]. Studies in mice revealed that SIRT3 expression was reduced in old aortic valves compared with young ones, signifying an age-associated SIRT3 reduction [31]. Overexpression of SIRT3 enhanced cells' ability to combat oxidative stress and reduced stress-mediated cell injury by activating catalase and MnSOD in human MSCs [32]. Another member of the sirtuin family, SIRT6, is a potential target for rejuvenation of aging stem cells [33]. A recent study demonstrated that knockdown of SIRT6 in human bone marrow MSCs resulted in impaired growth, proliferation, and migration ability, along with increased cell death and senescence [34]. Interestingly, recent results from our group showed that cytoglobin, a gene linked to oxidative stress and mitochondria respiration, promotes cardiac progenitor cell survival against oxidative stress via the upregulation of the $\mathrm{NF} \kappa \mathrm{B} /$ 




Figure 1: Summary of strategies used to rejuvenate aging stem cells and heal the injured heart. These methods result in an increase in proliferation and decrease in apoptosis and senescence, allowing for improved regeneration capabilities of the myocardium.

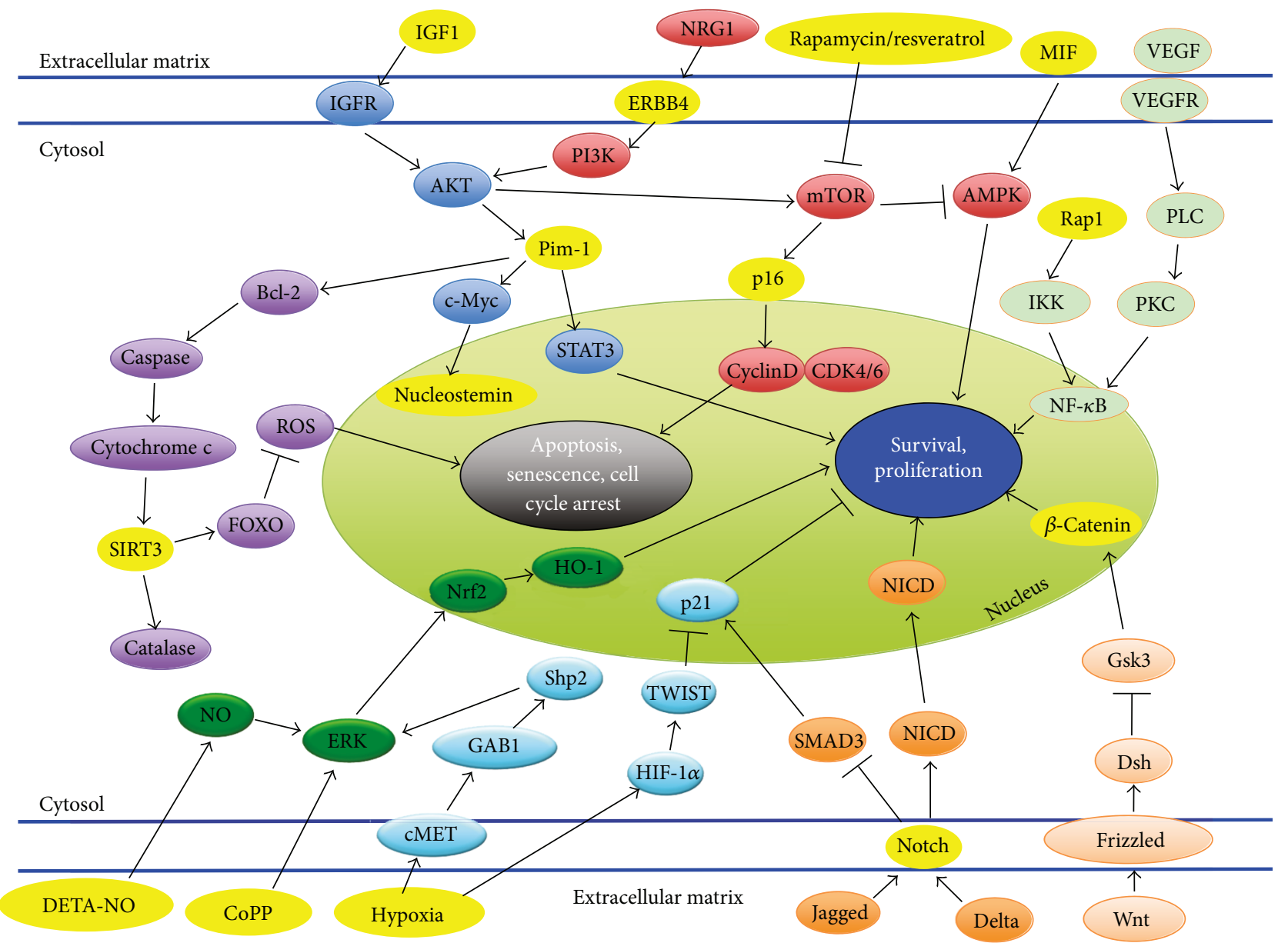

FIGURE 2: Molecular signaling pathways that are associated with rejuvenating aging stem/progenitor cells. Those that are currently being studied and were discussed in this review are highlighted in yellow. 
iNOS signal pathway and nitric oxide production, providing a novel molecular target that can be used to enhance the effectiveness of cardiac stem/progenitor cell therapy for ischemic heart disease [35]. Overall, mitochondria-associated proteins represent a potential target for rejuvenation of aging cardiac stem cells. However, future in vivo studies need to be done in order to show improved therapeutic effectiveness in the heart.

miRNAs in the heart are also significant contributors to disease; their altered expression may be partly responsible for cardiovascular disorders [36]. Differential expression of miRNAs in old cells compared to young cells, such as downregulation of miR-17, miR-19b, miR-20a, and miR-106a in aged cells, implicates miRNAs as markers of biological aging [37]. Recent work has found an increase in expression of a specific miRNA, miR-195, which targets telomerase reverse transcriptase (Tert) gene, causing deterioration of the regenerative ability of old MSCs. Transplantation of old MSCs with knockdown of miR-195 led to improvement in cardiac function and reduction of infarct size [38]. Another study has reported that downregulation of miR-29c in MSCs led to the suppression of both the p53-p21 and the p18-pRB pathways and abrogation of cellular senescence [39]. Another miRNA, miR-34a, is elevated in mouse hearts after myocardial infarction [40]. Overexpression of miR-34a is associated with increased apoptosis, lower viability, and increased senescence, possibly through activation of SIRT1/ FOXO3a pathway. Inhibition of miR-34a leads to fewer apoptotic cells and better viability [41] along with improved cardiac molecular signature and increased angiogenesis. Overall, miRNAs represent powerful therapeutic targets because they are small in length, around 22 nucleotides, and can be efficiently inhibited in vivo [42]. However, future work is needed to understand the mechanistic participation of various miRNAs in regulating cell senescence/aging.

Clinical trials, such as the SCIPIO phase 1, have supported the efficacy of CPC therapy [5]. However, ischemic heart disease is associated with increased age; therefore, it is necessary for cell-based therapies to reduce the harmful effects of aging. This can be done with genetic manipulation of the stem cells. Stem/progenitor cells can be extracted from a consenting patient through clinical methods and then isolated, cultured, and modified in vitro with genetic modification. The cells can be later transplanted back into the patient's own heart to assist in healing the damaged postinfarct myocardium [43]. Overall, Pim-1, nucleostemin, Rap1, ERBB4, SIRT3, SIRT6, and catalase along with various different miRNAs represent potential genes whose expression can be manipulated in order for stem cells to adopt a youthful phenotype. However, a limitation of genetic modification is that a combination of genes may need to be modified in order to effectively heal the aging infarcted heart. Overall, the fundamental goal in genetic modification is to eventually lead to an increase in proliferation and regenerative capacities of stem cells and inhibition of the senescent phenotype.

\section{Pharmaceutical Administration}

A variety of pathways (Figure 2) including the mTOR/PI3K, $\mathrm{WNT} / \beta$-catenin, ERK/NRF2, and STAT3/NF $\kappa \mathrm{B}$ can be targeted for rejuvenation by pharmacological manipulation. Drugs such as rapamycin can be used to rejuvenate aging cardiac stem cells. Rapamycin is an inhibitor of mammalian target of rapamycin (mTOR) [44], a major downstream component in the PI3K senescence pathway [45]. mTOR's inactivation by rapamycin inhibits the TORC1 complex [46] and brings cells from a senescent to a quiescent stage [47]. A recent study utilized rapamycin along with resveratrol, a drug known for activating AMPK, which increases mitochondrial biogenesis and function [48]. The combination of these drugs was found to modify the secretome of cardiac stem cells from explanted decompensated hearts (E-CSC) such that there was prevention of cardiomyocyte death and senescence. Additional investigations in which infarcted mice were injected with E-CSC treated with rapamycin and resveratrol showed improved cardiac output. Using drugs such as rapamycin and resveratrol has a high potential since it avoids the possible detrimental effects from genetic modification [49]. As with any drug, rapamycin has side effects, and future work is needed to expose whether side effects could limit its usage [44].

Another research group has identified the wingless integration (WNT) $/ \beta$-catenin pathway (Figure 2 ) as a potential target for rejuvenation of hMSCs used in stem cell therapy for cardiac repair [9]. The study showed that increased age was associated with reduced MSC proliferation, MSC differentiation, and $\mathrm{WNT} / \beta$-catenin signaling. However, some functions of MSCs from aging individuals could be revitalized with lithium therapy, which increases $\beta$-catenin availability to improve myogenic differentiation [50]. The $\mathrm{WNT} / \beta$-catenin pathway is closely related to stem cell renewal and differentiation through regulation of CTNNB1, which plays an essential role in cardiogenic development [9].

Targeting senescent cells is another possibility that can lead to rejuvenation through pharmaceutical administration. Most senescent cells express $\mathrm{p} 16^{\text {Ink4a }}$, a cyclin-dependent kinase inhibitor that leads to cell arrest by activating $\mathrm{Rb}$. The expression of $\mathrm{p} 16^{\text {Ink4a }}$ is also known to increase with aging (Figure 2). A novel transgene, INK-ATTAC, was used to eliminate $\mathrm{p} 16^{\text {Ink4a }}$-positive senescent cells upon administration of rosiglitazone to induce senescence. The administration and resulting clearance of senescent cells led to enhanced health span and a delay in multiple age-related phenotypes in progeroid mice [51]. Furthermore, senolytics, a new class of drugs that selectively kill senescent cells, represent a great potential for improving health span. Two drugs, dasatinib (D) and quercetin (Q), were successful in improving cardiac functioning in aging mice and reducing the number of senescent bone marrow-derived murine MSCs by interfering with the antiapoptotic and prosurvival mechanisms of senescent cells. The results indicate that senescent cells exert deleterious effects on cardiovascular function with aging, and that clearance of these cells represents a novel therapeutic approach for rejuvenation [52].

Pharmacological treatment with cobalt protoporphyrin (CoPP) is another method to improve therapeutic effectiveness of myocardial repair. CoPP is an inducer of heme oxygenase-1 (HO-1), which induces cellular protection. A previous study has shown that preconditioning of hCSCs with CoPP increases the resistance of these cells to oxidative 
stress-induced apoptosis via the activation of ERK/NRF2 signal pathways (Figure 2). Knockdown of HO-1 leads to a diminished cytokine effect, which suggests that the beneficial effects of CoPP preconditioning may be due to the secretion of protective cytokines [53]. Another study demonstrated that CoPP treatment in hypoxic cells reduced cell damage and increased the viability of cardiomyocytes by preserving mitochondrial membrane potential [54]. Furthermore, preconditioning hCSCs with CoPP leads to an increase in cell survival and proliferation after transplantation into the infarcted heart along with greater LV functional and structural improvement [55]. Overall, the HO-1 inducer, CoPP, is a promising candidate that can improve the efficiency of CSC-based therapies for ischemic heart disease. Nitric oxide (NO) is a gaseous signaling molecule shown to have an antiapoptotic role in many cells. Diethylenetriamine nitric oxide adduct (DETA-NO) is a chemical-based NO releaser. A recent study has demonstrated that preconditioning human cardiac stem cells with DETA-NO promotes cell survival and resistance to oxidative stress. Future work would require examination of the in vivo survival of hCSCs enhanced by DETA-NO preconditioning [56].

Pharmaceutical administration is a valid methodology for rejuvenation. Similar to the genetic modification technique, stem/progenitor cells can be extracted from a patient and cultured in vitro while being treated with pharmaceutical agents. However, a potential limitation of this strategy is that pharmaceutical agents can have a wide variety of effects, and therefore, a balance between side effects and benefits must be reached before the drugs can be used in a clinical setting. Perhaps, a combination of pharmacological treatment and genetic modification can be employed to rejuvenate aging stem cells, allowing for a more comprehensive approach.

\section{Optimization of Extracellular Factors}

The host environment plays a very important role in the outcome of cell therapy; allogenic cells from young, healthy donors have been used to overcome age-related stem cell dysfunction [57]. Furthermore, tissue aging is influenced by systemic and circulating factors. The detection of senescence factors by neighboring healthy cells might further progress cellular senescence, contributing to dysfunction associated with age-related cardiac diseases [2]. Factors that slow the age-dependent deterioration of the cell niche represent a new method for treating age-related diseases [58]. Using systemic and circulating factors to rejuvenate aging cells has been shown in nerve [58] and bone [59] cells. Heterochronic parabiosis experiments, in which a young and old mice are surgically linked so they develop a shared blood circulation [60], indicate that signals from a young circulation can impact the function of aging tissues [61].

Manipulation of the Notch pathway, in which ligands such as Delta interact with the Notch receptor (Figure 2), can be used to rejuvenate aging cardiac stem cells [62]. Studies have found that the failure of this pathway to be activated can lead to decline in the regenerative potential of muscle with age, due in part to impairment in upregulation of the Notch ligand Delta after muscle injury [63]. Using heterochronic parabiosis to restore Delta upregulation in aging satellite cells allows for enhanced activation and proliferation [64]. Notch activation has been found to restrain cardiac hypertrophy and fibrosis and promote cardiac precursor expansion. Communication between Jagged1-expressing cardiomyocytes and Notch-expressing MSCs is important to shift the response towards cardiac precursor expansion [65]. Activation of Notch signaling in the border zone after infarction promotes survival and improves cardiac function. Delivery of a peptide mimic of the Notch1 ligand Jagged 1 to the infarcted rat heart led to improvements in cardiac function and contractility [66]. Notch signaling also plays a crucial role in cell senescence; overexpression of Notch prolongs the lifespan of vascular endothelial cells by inhibiting a p16-dependent pathway [62]. Overall, manipulation of the Notch signaling pathway could be a new therapeutic target for treating ageassociated vascular diseases.

Another circulating factor implicated in cardiac disease is insulin-like growth factor 1 (IGF-1). IGF-1 is important in the recovery process of the heart because of its subsequent activation of PI3K/Akt signaling (Figure 2), which allows for enhanced cell survival, release of growth factors, stem cell mobilization, and angiogenesis. IGF-1 overexpression also accentuates the release of various growth factors, including HGF and VEGF, which contribute towards reduced cell apoptosis. Localized IGF-1 overexpression also significantly preserves LV wall thickness and contractile function in vivo [67]. A study has concluded that the IGF-1 receptor (IGF-1R) identifies a pool of human cardiac stem cells that have a superior therapeutic potential for myocardial regeneration. Overall, the presence of IGF-1R led to decreased apoptosis rates, enhanced telomerase activity, preserved telomere integrity, and favoring of hCSC division and survival. The expression of IGF-1R and the synthesis of IGF-1 are attenuated in aging hCSCs. Therefore, the study shows that a careful analysis of the phenotypic properties of the cells can be used to consider which cells are used for clinical treatment [68].

Many other cytokines and growth factors have been implicated as potential candidates for rejuvenation. Macrophage migration inhibitory factor (MIF) (Figure 2) is a cytokine that is released by ischemic cardiomyocytes in the heart, allowing for protection from injury and cellular apoptosis [69]. MIF-treated aged MSCs survived better than young MSCs not treated with MIF, suggesting that MIF possesses antiapoptotic properties. MIF also restored the trophic activity of MSCs as seen by the quantification of VEGF, HGF, and IGF levels [70]. Furthermore, tissue engineering has been used to seed angiogenic cytokines, VEGF and bFGF, onto a collagen scaffold. The cytokineenhanced, tissue-engineered patch rejuvenated aging cells, prolonged cell survival, and improved angiogenesis to restore ventricular function [71]. Gdf6, found to be downregulated in old MSCs, is another growth factor that may play a role in rejuvenation. A recent study in which Gdf6 was administered led to the restoration of the differentiation potential of aged MSCs in vitro and wielded beneficial effects on age-associated pathologies in mice [72]. An understanding of the mechanisms underlying the regenerative effects of various growth factors could lead to the 
TABLE 1: Advantages and disadvantages of the various strategies that were discussed.

Genetic modification

(i) Has stable effect

Advantages

(ii) Can directly target specific cell survival pathways

Disadvantages (i) May need multiple genes modified to have a significant effect
Pharmaceutical administration Optimization of extracellular factors

(i) Has the ability to effect multiple pathways at once

(ii) Can lead to release of growth factors and cytokines

(i) Has potential side effects (i) Takes into account the stem cell niche

(ii) Has potential to prime cells to endure the postinfarct environment

(i) Has multiple aspects of environment may need to be optimized to have significant effect development of novel therapeutic agents for the treatment of myocardial infarction.

Oxygen levels of stem cell niches are known to play a role in stem cell quiescence; changing oxygen concentrations can affect the survival and proliferation of stem cells when used for myocardial therapy. It has been shown that expansion of hCSCs under hypoxic conditions leads to greater engraftment and functional benefit after implantation into infarcted hearts of mice [73]. Further studies show that MSCs cultured in hypoxia activate the Akt and HGF-cMet signaling pathway (Figure 2), which leads to increase in migration rates. The culture in hypoxic conditions is also beneficial because it is more similar to the physiologic niche of MSCs in the bone marrow. A hind limb ischemia injury model was used to show that mice that received hypoxic preconditioned MSCs recovered faster than control groups [74]. A study with EPCs showed that hypoxia can prevent senescence, increase proliferation capacity and lifespan, and maintain the stem cell properties of EPCs through HIF1 $\alpha$-induced TWIST expression (Figure 2), which inhibits cell cycle arrest and replicative senescence [75]. Another study displayed a significant increase in the expression of prosurvival proteins such as NF- $\kappa \mathrm{B}$ and antiapoptotic proteins Bcl-2 and Blc-xL. Hypoxia-preconditioned MSC transplantation leads to enhanced angiogenesis and vascularization as compared to normal MSCs. Since $90 \%$ of grafted cells die within the first few days of transplantation, this protective effect of hypoxia is crucial to protect transplanted cells [6].

The identification of rejuvenating growth factors and environmental conditions opens new prospects to reverse the effects of cardiac aging. This strategy allows for preconditioning of the stem cells in order to optimize the extracellular environment before transplantation into the postinfarct heart. Altering systemic expression of factors that promote stem cell activity and culturing cells in hypoxic conditions are powerful methods of rejuvenation. However, the stem cell niche is very complex; therefore, further research is required before this strategy can lead to clinical translation.

\section{Other Strategies}

Other engineering strategies are being developed to rejuvenate the senescent heart. Two novel stem cell products have been engineered: CardioChimeras and CardioClusters. CardioChimeras are formed by fusing together CPCs with MSCs. Combined administration of hMSCs and human c-kit-positive CPCs into an infarcted heart has significantly improved myocardial structure and function [76]. This technique creates a cell hybrid that combines optimal traits such as proliferation, survival, and paracrine secretion. In addition, CardioClusters are comprised of a 3D microenvironment consisting of specific cell types isolated from the human heart such as CPCs, MSCs, EPCs, and fibroblasts. Furthermore, Cardiospheres are 3D spontaneous aggregations of heterogeneous stem cells and have been created to enhance communication between stem cells and the cardiac environment [9]. A clinical trial CADUCEUS has explored the effect of intracoronary infusion of cardiosphere-derived cells and found a significant reduction in scar mass [77]. CardioClusters, Cardiospheres, and CardioChimeras represent a next generation of stem cell therapy that can be used to rejuvenate the heart. However, these combinatorial cell therapies are limited because of differences in proliferation rates and survival after injection into the damaged heart [9]. More studies are required before this technique can be translated into clinical applications.

\section{Conclusion and Future Directions}

This review discusses the current strategies for the rejuvenation of aging stem cells in order to improve therapeutic techniques for myocardial repair. Cardiac stem cells have an innate ability to rejuvenate the myocardium; however, the aging population of patients has a compromised stem cell population in terms of functional capacity and regenerative potential [78]. Therefore, these aging stem cells must be rejuvenated by techniques such as the discussed methods of in vitro genetic modification, pharmaceutical administration, and optimization of extracellular factors. Recent studies show promising results of the ability of these techniques to rejuvenate the aging heart. However, more understanding of the combinatorial effects of these interventions and fine-tuning of these techniques, depicted in Figure 1, is required to evaluate the translational potential of these methods. Each strategy has its own advantages and disadvantages as outlined in Table 1. Overall, the success of myocardial regenerative treatment will require teamwork across various disciplines to make stem cell therapy a reliable method for cardiac repair.

\section{Conflicts of Interest}

The authors declare no potential conflicts of interest. 


\section{References}

[1] V. L. T. Ballard and J. M. Edelberg, "Stem cells and the regeneration of the aging cardiovascular system," Circulation Research, vol. 100, no. 8, pp. 1116-1127, 2007.

[2] A. Behfar and A. Terzic, "Stem cells versus senescence: the yin and yang of cardiac health," Journal of the American College of Cardiology, vol. 65, no. 2, pp. 148-150, 2015.

[3] S. Siddiqi and M. A. Sussman, "Cardiac hegemony of senescence," Current Translational Geriatrics and Experimental Gerontology Reports, vol. 2, no. 4, pp. 247-254, 2013.

[4] J. S. Forrester, A. J. White, S. Matsushita, T. Chakravarty, and R. R. Makkar, "New paradigms of myocardial regeneration post-infarction: tissue preservation, cell environment, and pluripotent cell sources," JACC Cardiovascular Interventions, vol. 2, no. 1, pp. 1-8, 2009.

[5] R. Bolli, A. R. Chugh, D. D'Amario et al., "Cardiac stem cells in patients with ischaemic cardiomyopathy (SCIPIO): initial results of a randomised phase 1 trial," The Lancet, vol. 378, no. 9806, pp. 1847-1857, 2011.

[6] X. Hu, S. P. Yu, J. L. Fraser et al., "Transplantation of hypoxiapreconditioned mesenchymal stem cells improves infarcted heart function via enhanced survival of implanted cells and angiogenesis," The Journal of Thoracic and Cardiovascular Surgery, vol. 135, no. 4, pp. 799-808, 2008.

[7] N. C. Ni, R. K. Li, and R. D. Weisel, "The promise and challenges of cardiac stem cell therapy," Seminars in Thoracic and Cardiovascular Surgery, vol. 26, no. 1, pp. 44-52, 2014.

[8] F. Paneni, C. Diaz Cañestro, P. Libby, T. F. Lüscher, and G. G. Camici, "The aging cardiovascular system: understanding it at the cellular and clinical levels," Journal of the American College of Cardiology, vol. 69, no. 15, pp. 1952-1967, 2017.

[9] N. Nguyen and M. A. Sussman, "Rejuvenating the senescent heart," Current Opinion in Cardiology, vol. 30, no. 3, pp. 235-239, 2015.

[10] M. A. Laflamme, J. Gold, C. Xu et al., "Formation of human myocardium in the rat heart from human embryonic stem cells," The American Journal of Pathology, vol. 167, no. 3, pp. 663-671, 2005.

[11] R. Liang and S. Ghaffari, "Stem cells, redox signaling, and stem cell aging," Antioxidants \& Redox Signaling, vol. 20, no. 12, pp. 1902-1916, 2014.

[12] C. Cencioni, F. Spallotta, F. Martelli et al., "Oxidative stress and epigenetic regulation in ageing and age-related diseases," International Journal of Molecular Sciences, vol. 14, no. 9, pp. 17643-17663, 2013.

[13] C. E. Murry, L. J. Field, and P. Menasche, "Cell-based cardiac repair: reflections at the 10-year point," Circulation, vol. 112, no. 20, pp. 3174-3183, 2005.

[14] P. Menasche, O. Alfieri, S. Janssens et al., "The myoblast autologous grafting in ischemic cardiomyopathy (MAGIC) trial: first randomized placebo-controlled study of myoblast transplantation," Circulation, vol. 117, no. 9, pp. 1189-1200, 2008.

[15] V. Schächinger, S. Erbs, A. Elsässer et al., "Intracoronary bone marrow-derived progenitor cells in acute myocardial infarction," The New England Journal of Medicine, vol. 355, no. 12, pp. 1210-1221, 2006.

[16] D. P. Del Re and J. Sadoshima, "Enhancing the potential of cardiac progenitor cells: pushing forward with Pim-1," Circulation Research, vol. 110, no. 9, pp. 1154-1156, 2012.
[17] K. M. Fischer, C. T. Cottage, W. Wu et al., "Enhancement of myocardial regeneration through genetic engineering of cardiac progenitor cells expressing Pim-1 kinase," Circulation, vol. 120, no. 21, pp. 2077-2087, 2009.

[18] S. Mohsin, M. Khan, J. Nguyen et al., "Rejuvenation of human cardiac progenitor cells with Pim-1 kinase," Circulation Research, vol. 113, no. 10, pp. 1169-1179, 2013.

[19] S. Mohsin, M. Khan, H. Toko et al., "Human cardiac progenitor cells engineered with Pim-I kinase enhance myocardial repair," Journal of the American College of Cardiology, vol. 60, no. 14, pp. 1278-1287, 2012.

[20] N. Hariharan, P. Quijada, S. Mohsin et al., "Nucleostemin rejuvenates cardiac progenitor cells and antagonizes myocardial aging," Journal of the American College of Cardiology, vol. 65, no. 2, pp. 133-147, 2015.

[21] K. Samse, J. Emathinger, N. Hariharan et al., "Functional effect of Pim1 depends upon intracellular localization in human cardiac progenitor cells," Journal of Biological Chemistry, vol. 290, no. 22, pp. 13935-13947, 2015.

[22] M. Gnecchi, H. He, O. D. Liang et al., "Paracrine action accounts for marked protection of ischemic heart by Aktmodified mesenchymal stem cells," Nature Medicine, vol. 11, no. 4, pp. 367-368, 2005.

[23] H. Teo, S. Ghosh, H. Luesch et al., "Telomere-independent Rap1 is an IKK adaptor and regulates NF- $\kappa \mathrm{B}$-dependent gene expression," Nature Cell Biology, vol. 12, no. 8, pp. 758-767, 2010.

[24] Y. Zhang, S. Chiu, X. Liang et al., "Rap1-mediated nuclear factor-kappaB (NF- $\kappa \mathrm{B})$ activity regulates the paracrine capacity of mesenchymal stem cells in heart repair following infarction," Cell Death Discovery, vol. 1, no. 1, p. 15007, 2015.

[25] K. Bersell, S. Arab, B. Haring, and B. Kühn, "Neuregulin1/ ErbB4 signaling induces cardiomyocyte proliferation and repair of heart injury," Cell, vol. 138, no. 2, pp. 257-270, 2009.

[26] X. Liang, Y. Ding, Y. Zhang et al., "Activation of NRG1-ERBB4 signaling potentiates mesenchymal stem cell-mediated myocardial repairs following myocardial infarction," Cell Death \& Disease, vol. 6, no. 5, p. e1765, 2015.

[27] N. Sun, R. J. Youle, and T. Finkel, “The mitochondrial basis of aging," Molecular Cell, vol. 61, no. 5, pp. 654-666, 2016.

[28] L. Mouchiroud, R. H. Houtkooper, N. Moullan et al., "The $\mathrm{NAD}^{+} /$sirtuin pathway modulates longevity through activation of mitochondrial UPR and FOXO signaling," Cell, vol. 154, no. 2, pp. 430-441, 2013.

[29] V. B. Pillai, N. R. Sundaresan, V. Jeevanandam, and M. P. Gupta, "Mitochondrial SIRT3 and heart disease," Cardiovascular Research, vol. 88, no. 2, pp. 250-256, 2010.

[30] N. R. Sundaresan, M. Gupta, G. Kim, S. B. Rajamohan, A. Isbatan, and M. P. Gupta, "Sirt3 blocks the cardiac hypertrophic response by augmenting Foxo3a-dependent antioxidant defense mechanisms in mice," The Journal of Clinical Investigation, vol. 119, no. 9, pp. 2758-2771, 2009.

[31] C. M. Roos, M. Hagler, B. Zhang, E. A. Oehler, A. Arghami, and J. D. Miller, "Transcriptional and phenotypic changes in aorta and aortic valve with aging and MnSOD deficiency in mice," American Journal of Physiology Heart and Circulatory Physiology, vol. 305, no. 10, pp. H1428-H1439, 2013.

[32] X. Q. Wang, Y. Shao, C. Y. Ma et al., "Decreased SIRT3 in aged human mesenchymal stromal/stem cells increases cellular susceptibility to oxidative stress," Journal of Cellular and Molecular Medicine, vol. 18, no. 11, pp. 2298-2310, 2014. 
[33] R. Mostoslavsky, K. F. Chua, D. B. Lombard et al., "Genomic instability and aging-like phenotype in the absence of mammalian SIRT6," Cell, vol. 124, no. 2, pp. 315-329, 2006.

[34] X. Y.Zhai, P. Yan, J. Zhang et al., "Knockdown of SIRT6 enables human bone marrow mesenchymal stem cell senescence," Rejuvenation Research, vol. 19, no. 5, pp. 373-384, 2016.

[35] S. Zhang, X. Li, F. L. Jourd'heuil et al., "Cytoglobin promotes cardiac progenitor cell survival against oxidative stress via the upregulation of the $\mathrm{NF} \kappa \mathrm{B} / \mathrm{iNOS}$ signal pathway and nitric oxide production," Scientific Reports, vol. 7, no. 1, article 10754, 2017.

[36] E. M. Small and E. N. Olson, "Pervasive roles of microRNAs in cardiovascular biology," Nature, vol. 469, no. 7330, pp. 336-342, 2011.

[37] M. Hackl, S. Brunner, K. Fortschegger et al., "miR-17, miR19b, miR-20a, and miR-106a are down-regulated in human aging," Aging Cell, vol. 9, no. 2, pp. 291-296, 2010.

[38] M. Okada, H. W. Kim, K. Matsu-ura, Y. G. Wang, M. Xu, and M. Ashraf, "Abrogation of age-induced microRNA195 rejuvenates the senescent mesenchymal stem cells by reactivating telomerase," Stem Cells, vol. 34, no. 1, pp. 148-159, 2016.

[39] J. Shang, Y. Yao, X. Fan et al., "miR-29c-3p promotes senescence of human mesenchymal stem cells by targeting CNOT6 through p53-p21 and p16-pRB pathways," Biochimica et Biophysica Acta (BBA) - Molecular Cell Research, vol. 1863, no. 4, pp. 520-532, 2016.

[40] R. C. Y. Lin, K. L. Weeks, X. M. Gao et al., "PI3K(p110 $\alpha)$ protects against myocardial infarction-induced heart failure: identification of PI3K-regulated miRNA and mRNA," Arteriosclerosis, Thrombosis, and Vascular Biology, vol. 30, no. 4, pp. 724-732, 2010.

[41] F. Zhang, J. Cui, X. Liu et al., "Roles of microRNA-34a targeting SIRT1 in mesenchymal stem cells," Stem Cell Research \& Therapy, vol. 6, no. 1, p. 195, 2015.

[42] B. C. Bernardo, X. M. Gao, C. E. Winbanks et al., "Therapeutic inhibition of the miR-34 family attenuates pathological cardiac remodeling and improves heart function," Proceedings of the National Academy of Sciences of the United States of America, vol. 109, no. 43, pp. 17615-17620, 2012.

[43] R. Khatiwala and C. Cai, "Strategies to enhance the effectiveness of adult stem cell therapy for ischemic heart diseases affecting the elderly patients," Stem Cell Reviews and Reports, vol. 12, no. 2, pp. 214-223, 2016.

[44] M. V. Blagosklonny, “Aging, stem cells, and mammalian target of rapamycin: a prospect of pharmacologic rejuvenation of aging stem cells," Rejuvenation Research, vol. 11, no. 4, pp. 801-808, 2008.

[45] X. M. Gao, G. Wong, B. Wang et al., "Inhibition of mTOR reduces chronic pressure-overload cardiac hypertrophy and fibrosis," Journal of Hypertension, vol. 24, no. 8, pp. 16631670, 2006.

[46] R. Zoncu, A. Efeyan, and D. M. Sabatini, "mTOR: from growth signal integration to cancer, diabetes and ageing," Nature Reviews Molecular Cell Biology, vol. 12, no. 1, pp. 21-35, 2011.

[47] L. G. Korotchkina, O. V. Leontieva, E. I. Bukreeva, Z. N. Demidenko, A. V. Gudkov, and M. V. Blagosklonny, "The choice between p53-induced senescence and quiescence is determined in part by the mTOR pathway," Aging Cell, vol. 2, no. 6, pp. 344-352, 2010.
[48] S. J. Park, F. Ahmad, A. Philp et al., "Resveratrol ameliorates aging-related metabolic phenotypes by inhibiting cAMP phosphodiesterases," Cell, vol. 148, no. 3, pp. 421-433, 2012.

[49] E. Avolio, G. Gianfranceschi, D. Cesselli et al., "Ex vivo molecular rejuvenation improves the therapeutic activity of senescent human cardiac stem cells in a mouse model of myocardial infarction," Stem Cells, vol. 32, no. 9, pp. 2373-2385, 2014.

[50] K. R. Brunt, Y. Zhang, A. Mihic et al., "Role of WNT/ $\beta$-catenin signaling in rejuvenating myogenic differentiation of aged mesenchymal stem cells from cardiac patients," The American Journal of Pathology, vol. 181, no. 6, pp. 2067-2078, 2012.

[51] D. J. Baker, T. Wijshake, T. Tchkonia et al., "Clearance of p16 ${ }^{\text {Ink4a }}$-positive senescent cells delays ageing-associated disorders," Nature, vol. 479, no. 7372, pp. 232-236, 2011.

[52] Y. Zhu, T. Tchkonia, T. Pirtskhalava et al., "The Achilles' heel of senescent cells: from transcriptome to senolytic drugs," Aging Cell, vol. 14, no. 4, pp. 644-658, 2015.

[53] C. Cai, L. Teng, D. Vu et al., "The heme oxygenase 1 inducer (CoPP) protects human cardiac stem cells against apoptosis through activation of the extracellular signal-regulated kinase (ERK)/NRF2 signaling pathway and cytokine release," Journal of Biological Chemistry, vol. 287, no. 40, pp.33720-33732, 2012.

[54] Y. Issan, R. Kornowski, D. Aravot et al., "Heme oxygenase-1 induction improves cardiac function following myocardial ischemia by reducing oxidative stress," PLoS One, vol. 9, no. 3, article e92246, 2014.

[55] C. Cai, Y. Guo, L. Teng et al., "Preconditioning human cardiac stem cells with an HO-1 inducer exerts beneficial effects after cell transplantation in the infarcted murine heart," Stem Cells, vol. 33, no. 12, pp. 3596-3607, 2015.

[56] L. Teng, E. Bennett, and C. Cai, "Preconditioning c-Kitpositive human cardiac stem cells with a nitric oxide donor enhances cell survival through activation of survival signaling pathways," Journal of Biological Chemistry, vol. 291, no. 18, pp. 9733-9747, 2016.

[57] A. Ludke, R. K. Li, and R. D. Weisel, "The rejuvenation of aged stem cells for cardiac repair," Canadian Journal of Cardiology, vol. 30, no. 11, pp. 1299-1306, 2014.

[58] L. Katsimpardi, N. K. Litterman, P. A. Schein et al., "Vascular and neurogenic rejuvenation of the aging mouse brain by young systemic factors," Science, vol. 344, no. 6184, pp. 630-634, 2014.

[59] G. S. Baht, D. Silkstone, L. Vi et al., "Exposure to a youthful circulation rejuvenates bone repair through modulation of $\beta$-catenin," Nature Communications, vol. 6, no. 1, p. 7131, 2015.

[60] A. R. Mendelsohn and J. W. Larrick, "Rejuvenation of aging hearts," Rejuvenation Research, vol. 16, no. 4, pp. 330-332, 2013.

[61] F. S. Loffredo, M. L. Steinhauser, S. M. Jay et al., "Growth differentiation factor 11 is a circulating factor that reverses age-related cardiac hypertrophy," Cell, vol. 153, no. 4, pp. 828-839, 2013.

[62] Y. Yoshida, Y. Hayashi, M. Suda et al., "Notch signaling regulates the lifespan of vascular endothelial cells via a p16dependent pathway," PLoS One, vol. 9, no. 6, article e100359, 2014.

[63] M. E. Carlson, C. Suetta, M. J. Conboy et al., "Molecular aging and rejuvenation of human muscle stem cells," EMBO Molecular Medicine, vol. 1, no. 8-9, pp. 381-391, 2009.

[64] I. M. Conboy, M. J. Conboy, A. J. Wagers, E. R. Girma, I. L. Weissman, and T. A. Rando, "Rejuvenation of aged progenitor 
cells by exposure to a young systemic environment," Nature, vol. 433, no. 7027, pp. 760-764, 2005.

[65] M. Nemir, M. Metrich, I. Plaisance et al., "The Notch pathway controls fibrotic and regenerative repair in the adult heart," European Heart Journal, vol. 35, no. 32, pp. 2174-2185, 2014.

[66] A. V. Boopathy, M. D. Martinez, A. W. Smith, M. E. Brown, A. J. García, and M. E. Davis, "Intramyocardial delivery of Notch ligand-containing hydrogels improves cardiac function and angiogenesis following infarction," Tissue Engineering Part A, vol. 21, no. 17-18, pp. 2315-2322, 2015.

[67] H. K. Haider, S. Jiang, N. M. Idris, and M. Ashraf, "IGF-1overexpressing mesenchymal stem cells accelerate bone marrow stem cell mobilization via paracrine activation of SDF- $1 \alpha /$ CXCR4 signaling to promote myocardial repair," Circulation Research, vol. 103, no. 11, pp. 1300-1308, 2008.

[68] D. D'Amario, M. C. Cabral-da-Silva, H. Zheng et al., "Insulin-like growth factor-1 receptor identifies a pool of human cardiac stem cells with superior therapeutic potential for myocardial regeneration," Circulation Research, vol. 108, no. 12, pp. 1467-1481, 2011.

[69] E. J. Miller, J. Li, L. Leng et al., "Macrophage migration inhibitory factor stimulates AMP-activated protein kinase in the ischaemic heart," Nature, vol. 451, no. 7178, pp. 578-582, 2008.

[70] W. Xia, F. Zhang, C. Xie, M. Jiang, and M. Hou, "Macrophage migration inhibitory factor confers resistance to senescence through CD74-dependent AMPK-FOXO3a signaling in mesenchymal stem cells," Stem Cell Research \& Therapy, vol. 6, no. 1, p. 82, 2015.

[71] K. Kang, L. Sun, Y. Xiao et al., "Aged human cells rejuvenated by cytokine enhancement of biomaterials for surgical ventricular restoration," Journal of the American College of Cardiology, vol. 60, no. 21, pp. 2237-2249, 2012.

[72] D. Hisamatsu, M. Ohno-Oishi, S. Nakamura, Y. Mabuchi, and H. Naka-Kaneda, "Growth differentiation factor 6 derived from mesenchymal stem/stromal cells reduces age-related functional deterioration in multiple tissues," Aging, vol. 8, no. 6, pp. 1259-1275, 2016.

[73] T. S. Li, K. Cheng, K. Malliaras et al., "Expansion of human cardiac stem cells in physiological oxygen improves cell production efficiency and potency for myocardial repair," Cardiovascular Research, vol. 89, no. 1, pp. 157-165, 2011.

[74] I. Rosová, M. Dao, B. Capoccia, D. Link, and J. A. Nolta, “Hypoxic preconditioning results in increased motility and improved therapeutic potential of human mesenchymal stem cells," Stem Cells, vol. 26, no. 8, pp. 2173-2182, 2008.

[75] S. H. Lee, J. H. Lee, S. Y. Yoo, J. Hur, H. S. Kim, and S. M. Kwon, "Hypoxia inhibits cellular senescence to restore the therapeutic potential of old human endothelial progenitor cells via the hypoxia-inducible factor- $1 \alpha$-TWIST-p21 axis," Arteriosclerosis, Thrombosis, and Vascular Biology, vol. 33, no. 10, pp. 2407-2414, 2013.

[76] P. Quijada, H. T. Salunga, N. Hariharan et al., "Cardiac stem cell hybrids enhance myocardial repair," Circulation Research, vol. 117, no. 8, pp. 695-706, 2015.

[77] D. Milasinovic and W. Mohl, "Contemporary perspective on endogenous myocardial regeneration," World Journal of Stem Cells, vol. 7, no. 5, pp. 793-805, 2015.

[78] S. Siddiqi and M. A. Sussman, "Cell and gene therapy for severe heart failure patients: the time and place for Pim-1 kinase," Expert Review of Cardiovascular Therapy, vol. 11, no. 8, pp. 949-957, 2013. 


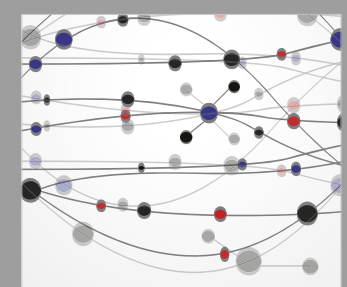

The Scientific World Journal
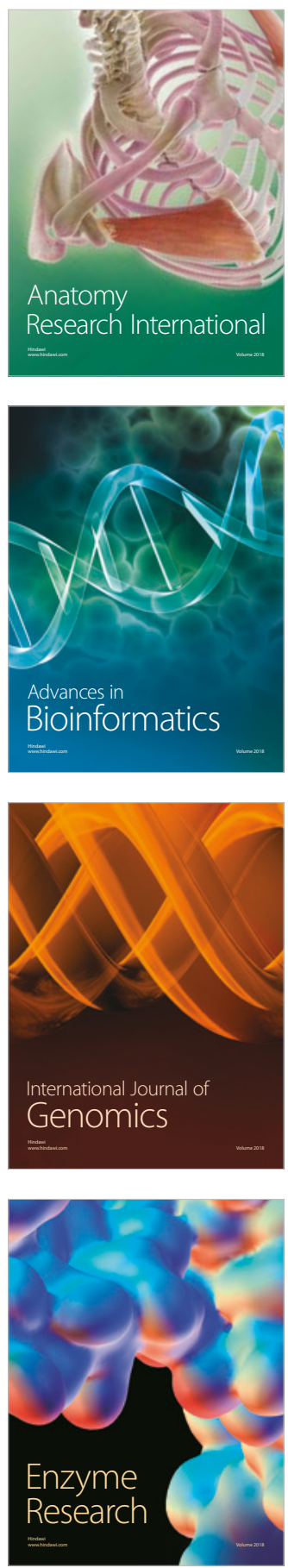
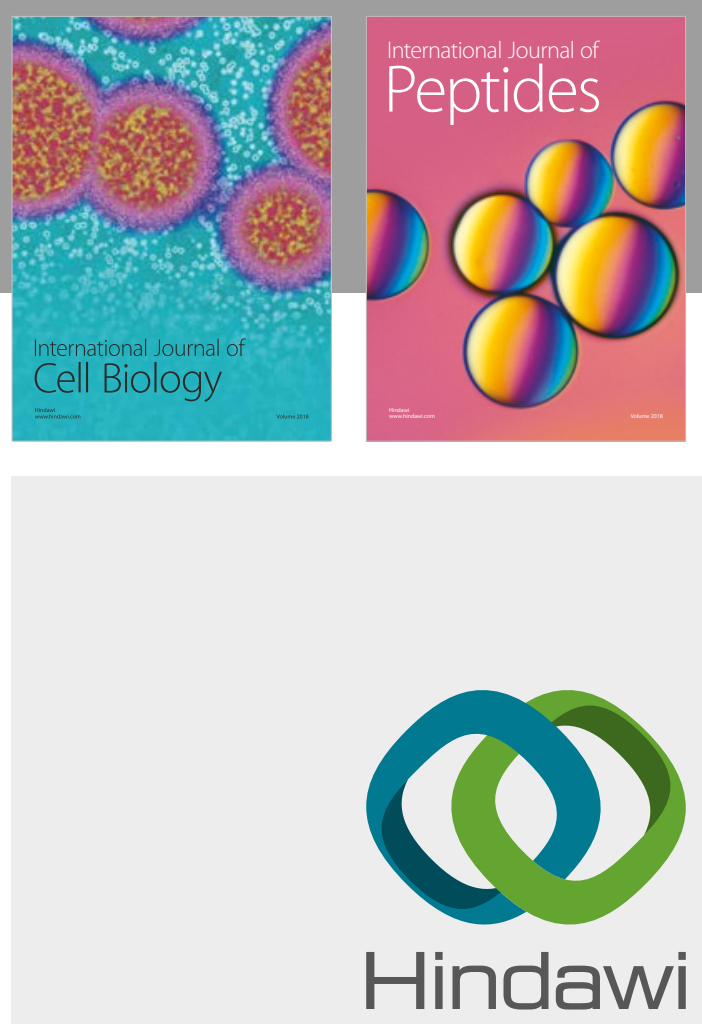

Submit your manuscripts at

www.hindawi.com
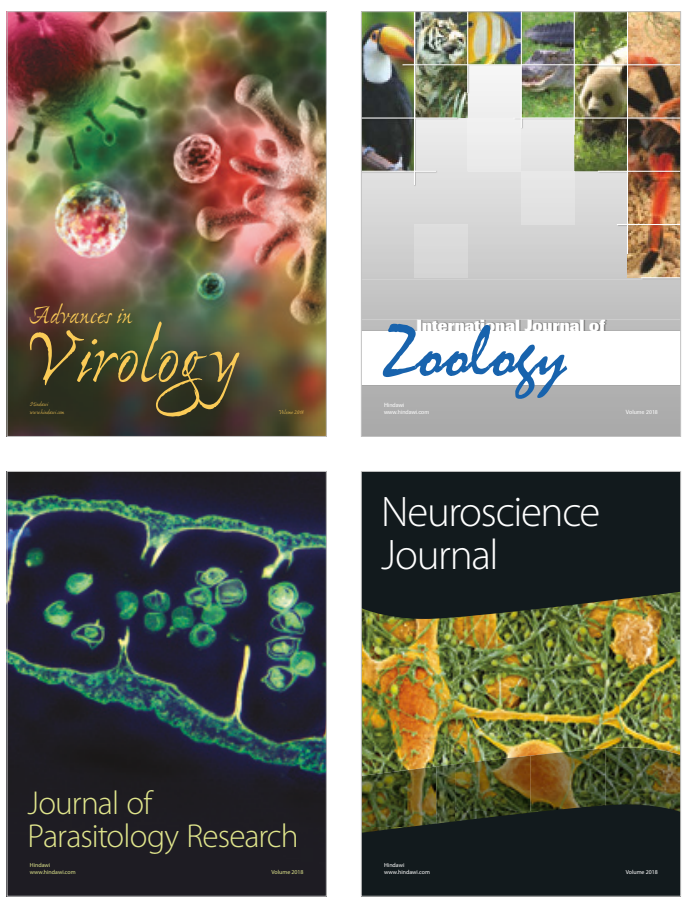
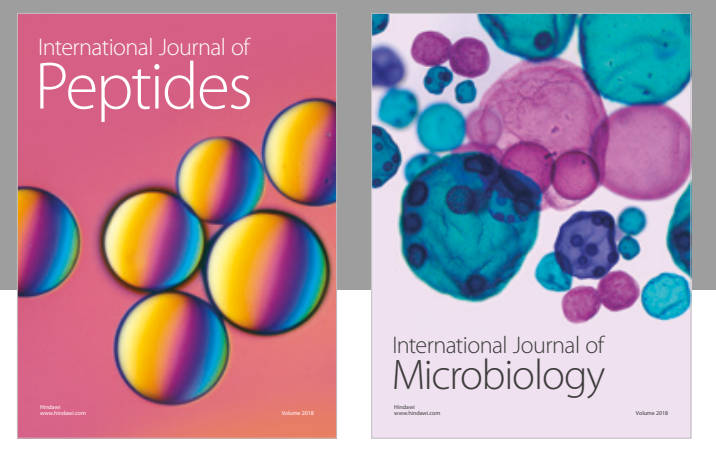

nternational Journal of Microbiology
Journal of
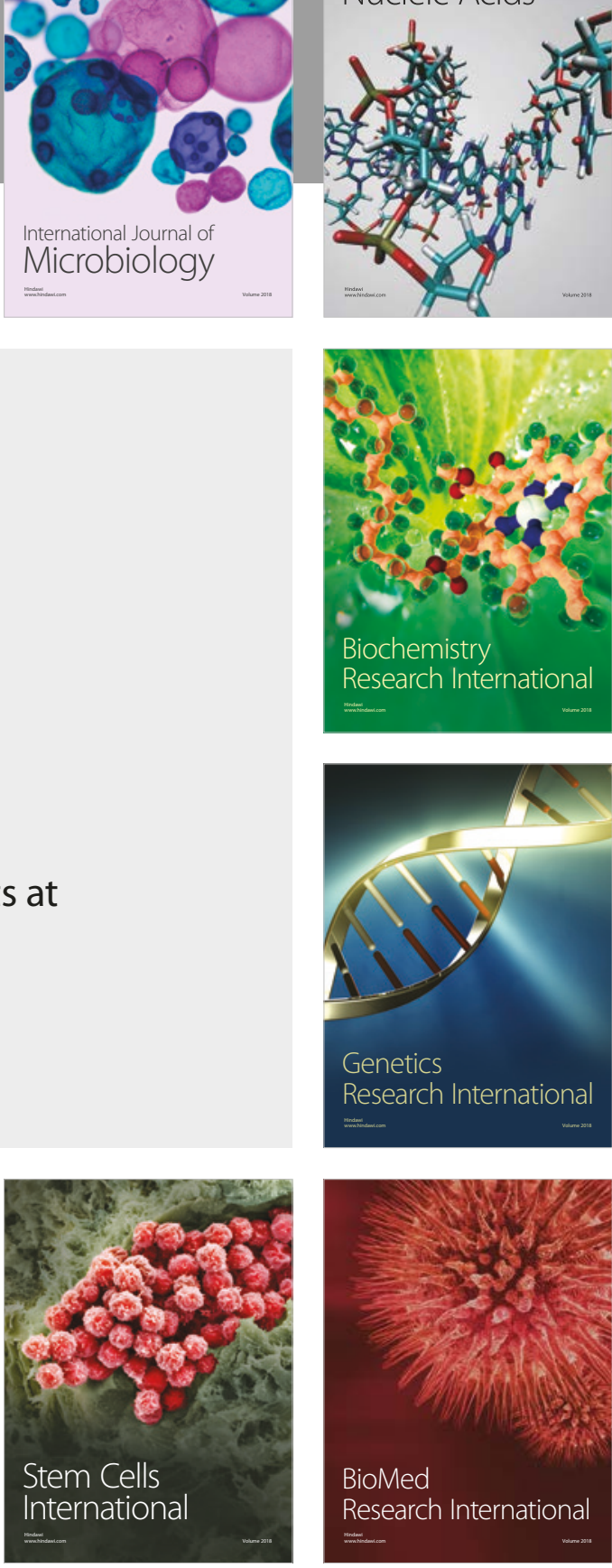
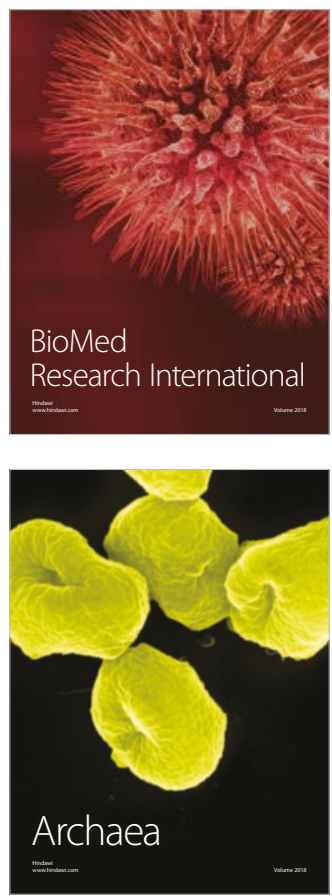